\title{
Beamlet scraping and its influence on the beam divergence at the BATMAN Upgrade test facility
}

\author{
C. Wimmer, ${ }^{1, \text { a) }}$ F. Bonomo, ${ }^{1}$ A. Hurlbatt, ${ }^{1}$ L. Schiesko, ${ }^{1}$ U. Fantz, ${ }^{1}$ N. den Harder, ${ }^{1}$ B. Heinemann, ${ }^{1}$ A. Mimo, ${ }^{1}$ \\ G. Orozco, ${ }^{1}$ M. Agostini, ${ }^{2}$ M. Barbisan, ${ }^{2}$ M. Brombin, ${ }^{2}$ R. Delogu, ${ }^{2}$ A. Pimazzoni, ${ }^{2}$ C. Poggi, ${ }^{2}$ G. Serianni, ${ }^{2}$ M. \\ Ugoletti, ${ }^{2}$ and P. Veltri ${ }^{3}$ \\ 1) Max-Planck-Institut für Plasmaphysik (IPP), Boltzmannstr. 2, 85748 Garching, Germany \\ 2) Consorzio RFX, Corso Stati Uniti 4, 35127 Padova, Italy \\ 3) ITER Organization, Route de Vinon sur Verdon, CS 90 046, 13067 St. Paul-lez-Durance, \\ France
}

(Dated: 12 December 2019)

For the ITER fusion experiment, two neutral beam injectors are required for plasma heating and current drive. Each injector supplies a power of about $17 \mathrm{MW}$, obtained from neutralization of $40 \mathrm{~A}(46 \mathrm{~A}), 1 \mathrm{MeV}(0.87 \mathrm{MeV})$ negative deuterium (hydrogen) ions. The full beam is composed of 1280 beamlets, formed in 16 beamlet groups and strict requirements apply to the beamlet core divergence $(<7 \mathrm{mrad})$. The test facility BATMAN Upgrade uses an ITER-like grid with one beamlet group, which consists of 70 apertures.

In a joint campaign performed by IPP and Consorzio RFX to better assess the beam optics, the divergence of a single beamlet was compared to a group of beamlets at BATMAN Upgrade. The single beamlet is measured by a CFC tile calorimeter and Beam Emission Spectroscopy, whereas the divergence of the group of beamlets is measured with Beam Emission Spectroscopy only. When increasing the RF power at low extraction voltages, the divergence of the beamlet and of the group of beamlets is continuously decreasing and no inflection point towards an overperveant beam is found. At the same time, scraping of the extracted ion beam at the second grid (extraction grid) takes place at higher RF power, supported by the absence of the normally seen linear behavior between the measured negative ion density in the plasma close to the extraction system and the measured extracted ion current. Beside its influence on the divergence, the beamlet scraping needs to be considered for the determination of the correct perveance and contributes to the measured co-extracted electron current.

\section{INTRODUCTION}

Neutral Beam Injection (NBI) is an essential component for the upcoming ITER fusion device used for plasma heating and current drive. Two beam lines with a total heating power of $33 \mathrm{MW}$ are foreseen, and each one needs to supply a beam of deuterium (or hydrogen) atoms with an energy of $1 \mathrm{MeV}$ $(870 \mathrm{keV})^{1,2}$. The beam of each beamline is composed out of 1280 individual beamlets, extracted and accelerated from an ion source with a grid system containing 1280 apertures arranged in 16 aperture groups of 80 apertures each. In order to minimize transmission losses in the beam duct at ITER, strict requirements apply on the beam divergence (the core divergence of each beamlet needs to be $<7 \mathrm{mrad}^{2,3}$ ).

As the neutralization efficiency of positive ions in the used gas neutralizer is almost zero at the required particle energies $^{4}$, the ITER NBI requires a source of negative deuterium (hydrogen) ions. The ion source for ITER is based on a modular design ${ }^{3,5}$; BATMAN Upgrade ${ }^{6}$ (BUG) is a test facility with an ion source $\approx 1 / 8$ the size of the ITER source, powered by one RF driver ( $1 \mathrm{MHz}$, up to $90 \mathrm{~kW}$ power), and contributes to the $R \& D$ roadmap towards the ITER source. $\mathrm{Cs}$ is evaporated into the source to enable surface production of negative ions due to the low work function of caesiated surfaces ${ }^{7}$. The total available high voltage that can be applied to the source for beam extraction and accelera-

\footnotetext{
a)Electronic mail: christian.wimmer@ipp.mpg.de.
}

tion at BATMAN Upgrade is $-45 \mathrm{kV}$. To extract ion current densities in the range of the ITER requirements (these are $j_{\mathrm{D}^{-}}=28.6 \mathrm{~mA} / \mathrm{cm}^{2}$ or $j_{\mathrm{H}^{-}}=32.9 \mathrm{~mA} / \mathrm{cm}^{2}$ ), an extraction voltage $U_{\text {ex }}$ of $10 \mathrm{kV}$ is applied, this being the voltage between the plasma-facing Plasma Grid (PG) and the second grid, Extraction Grid (EG). However, the remaining acceleration voltage $U_{\text {acc }}$ of $35 \mathrm{kV}$ is then not sufficient to achieve a low-divergent beam (a ratio of 6-7 is required for $U_{\mathrm{acc}} / U_{\mathrm{ex}}$ to achieve the divergence minimum of the beamlets at BATMAN Upgrade ${ }^{8}$ ). Thus, for studies of the beam divergence, a lower extraction voltage (typically $U_{\mathrm{ex}}=4-5 \mathrm{kV}$ ) and consequently a lower beam current to keep the beam at optimum perveance needs to be used.

Beam Emission Spectroscopy (BES) ${ }^{5,9,10}$ is regularly used at BATMAN Upgrade and other test facilities for the measurement of the beam divergence. BES measures an integrated signal along its line-of-sight covering the beam composed of the overlap of several beamlets. A minimum divergence of $\approx 20 \mathrm{mrad}$ is found for the beam at BATMAN Upgrade, whereas simulations of a single beamlet using the IBSimu code $^{11}$ predict a minimum divergence of about $11-14 \mathrm{mrad}$ (i.e. $\left.0.6-0.8^{\circ}\right)^{12,13}$. The minimum divergence depends on the temperature of the negative ions through their initial velocity perpendicular to the beam direction. An ion temperature of $1 \mathrm{eV}$ results in a minimum divergence in the given range. In order to better assess the divergence disagreement between measurement and simulation, a joint campaign between IPP and Consorzio RFX has been carried out recently, in which the difference in the divergence of a single beamlet and a group of beamlets ( 35 apertures) has been investigated 


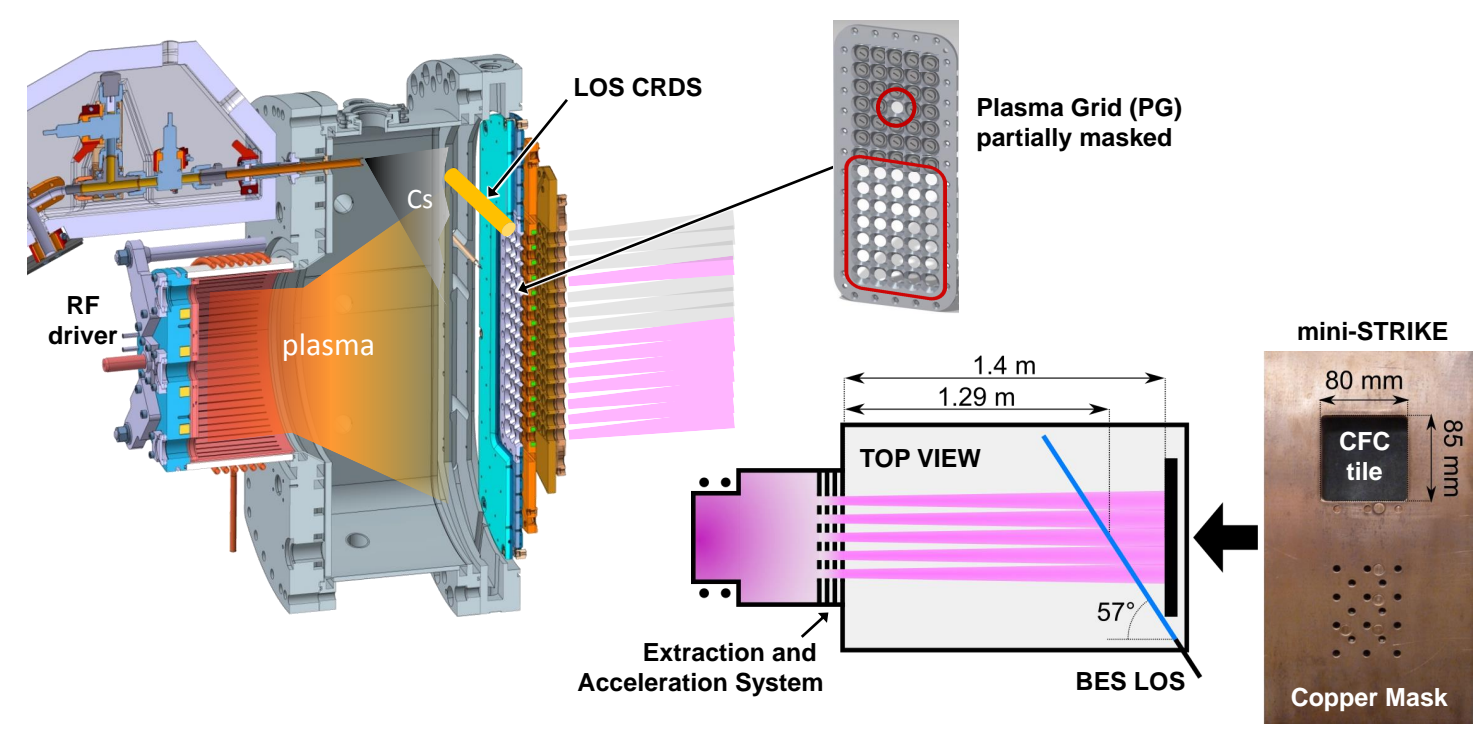

FIG. 1. Left: vertical cut through the ion source of BATMAN Upgrade, showing the position of the CRDS LOS. Top right: CAD drawing of the masked PG. Bottom: Top view of the ion source and the drift region of the beam with the positions of the BES lines of sight and the mini-STRIKE calorimeter indicated. Bottom right: front view of the mini-STRIKE calorimeter, showing the CFC tile for the single beamlet through the cutted copper mask.

at BATMAN Upgrade using two beam diagnostics ${ }^{8}$ : the miniSTRIKE CFC tile calorimeter ${ }^{14,15}$ (supplied and operated by Consorzio RFX) is used for determination of the divergence of the single beamlet; BES measures the divergence of both the single beamlet and the group of beamlets by two lines of sight. Excellent agreement has been shown between the two diagnostics for the single beamlet, with the divergence in agreement to beamlet simulations. The group of beamlets showed an increased divergence (up to a factor of two higher), being mainly attributed to the non-compensated row-wise zig-zag deflection at BATMAN Upgrade caused by electron deflection magnets (see section II) and the mixing of beamlets in the BES signal ${ }^{8}$.

At low extraction voltage $\left(U_{\text {ex }} \lesssim 5 \mathrm{kV}\right)$, it is observed that increasing the RF power $P_{\mathrm{RF}}$ and hence the density of negative ions in the plasma close to the extraction system leads to a saturation of the extracted ion current from a certain level of RF power; this effect is stronger for lower $U_{\mathrm{ex}}$ and is, in contrast, not observed for higher applied extraction voltage ${ }^{16}$. The cause for this effect and its influence on the divergence of the ion beam are presented in this paper.

\section{SETUP AT BATMAN UPGRADE}

The setup of the applied diagnostics at the BATMAN Upgrade test facility is shown in Figure 1. BATMAN Upgrade is equipped with a four-grid extraction and acceleration system, the Plasma Grid (PG), Extraction Grid (EG), Repeller Grid (RG) and Grounded Grid (GG). In this campaign, the RG has been kept at the same (ground) potential as the GG. Permanent magnets are embedded in the EG for deflecting co- extracted electrons out of the extracted particle beam; their row-wise changing polarity leads to a horizontal zig-zag deflection of the extracted ion beam. For extraction of a single beamlet, most of the apertures (normally 70 apertures with a diameter of $14 \mathrm{~mm}$ ) have been blocked on the top part of the PG, leaving just an isolated aperture open; the bottom part has been kept fully open (35 apertures). Thus, a single beamlet is formed in the top part of the extraction system, whereas a group of beamlets is formed in the bottom part. The BES02 system at BATMAN Upgrade consists of 11 vertically arranged ( $3 \mathrm{~cm}$ spacing) horizontal lines of sight (LOS), intercepting the beam at a distance of $1.29 \mathrm{~m}$ downstream from the $\mathrm{GG}^{6}$. Two BES02 LOS are used to determine the divergence of the single beamlet and grouped beamlets, respectively ${ }^{8}$. The divergence is calculated from the width of a Gaussian fit to the measured Doppler-shifted $\mathrm{H}_{\alpha}$ radiation ${ }^{9,17}$.

The mini-STRIKE CFC calorimeter is placed at a position $1.4 \mathrm{~m}$ downstream the GG; a CFC tile is positioned in a way that it intercepts the single beamlet. The footprint is recorded with an IR camera (FLIR SC655) from the backside of the CFC tile; the divergence is then calculated from the width of a 2D Gaussian fit of the beamlet footprint. More details on the two beam diagnostics and their evaluation are given elsewhere ${ }^{8}$.

Cavity Ring-Down Spectroscopy (CRDS) is used to determine the LOS-averaged density of negative hydrogen ions in the plasma close to the extraction system at a horizontal LOS (length: $0.2 \mathrm{~m}$ ) in the top part of the source with a distance of $2.7 \mathrm{~cm}$ upstream to the $\mathrm{PG}^{5,18}$.

The extracted currents are measured in the following way: the drain current $I_{\text {drain }}$ is the total current drawn from the HV system, thus containing all charges (negative ions and 

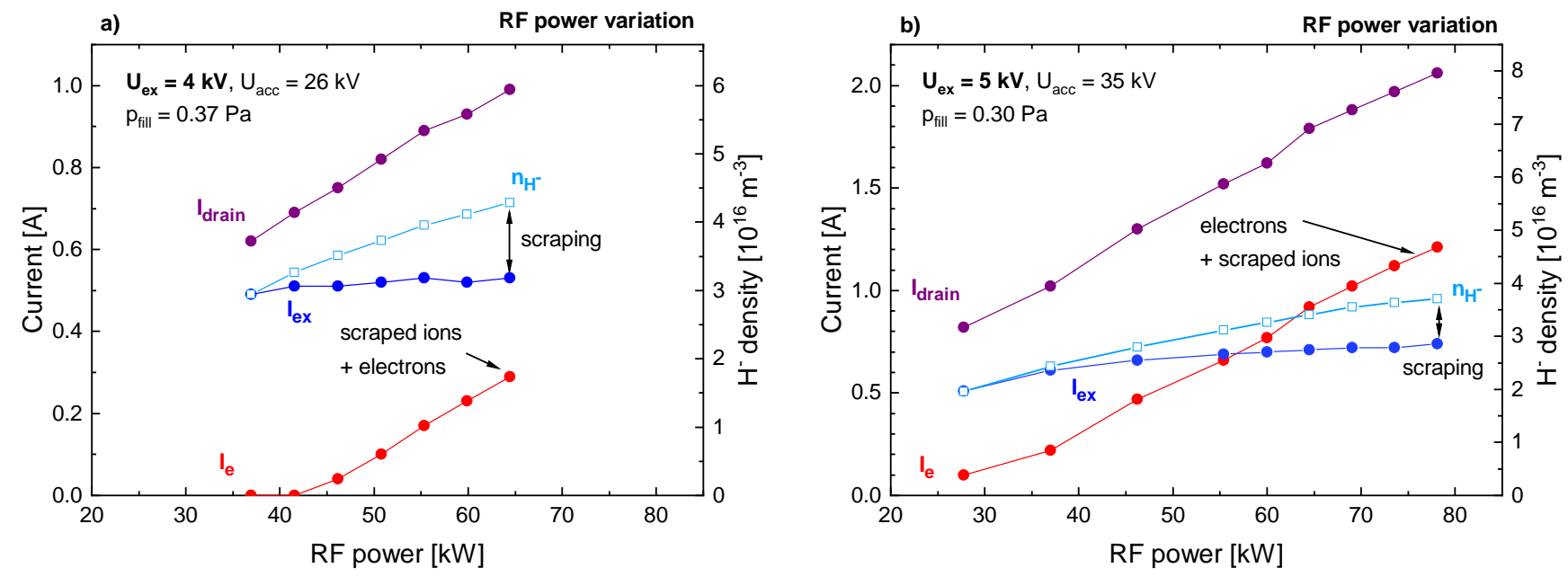

FIG. 2. Extracted currents (ion and electron), drain current, and density of negative ions as function of the applied RF power at a) $4 \mathrm{kV}$ extraction voltage and b) $5 \mathrm{kV}$ extraction voltage.

electrons) that pass the PG. The extracted ion current $I_{\mathrm{ex}}$ is measured as the current on ground potential, containing all charges that pass the EG. The co-extracted electron current $I_{\mathrm{e}}$ is measured as the current on the EG. Thus, if negative ions are scraped at the EG, they are counted as electrons. The extracted $\mathrm{H}^{-}$current density $j_{\mathrm{ex}}$ is calculated using $I_{\mathrm{ex}}\left(j_{\mathrm{ex}}=I_{\mathrm{ex}} / A_{\mathrm{ex}}\right.$, $A_{\text {ex }}$ is the area of open apertures in the PG, i.e. $55.4 \mathrm{~cm}^{2}$ in the present setup). Details on the grid system and the electrical circuit can be found elsewhere ${ }^{5,19}$. It should be noted that the measured drain current is not exactly the sum of the measured ion and electron current. This deviation is explained by the measurement uncertainties of the used current transducers, which are of greater impact at the relatively low currents resulting from the present setup (masked PG and low extraction voltages).

A more detailled description of BATMAN Upgrade can be found elsewhere ${ }^{6,19,20}$. All measurements in the campaign shown in this paper have been carried out in hydrogen operation.

\section{RESULTS}

\section{A. Evidence of Beamlet Scraping}

Shown in Figure 2 are the extracted currents $\left(I_{\mathrm{ex}}\right.$ and $\left.I_{\mathrm{e}}\right)$, the drain current $I_{\text {drain }}$ as well as the $\mathrm{H}^{-}$density in the plasma as function of the applied RF power for two cases: an extraction voltage of $4 \mathrm{kV}$ and a source filling pressure of $0.37 \mathrm{~Pa}$ has been used in a), whereas a higher extraction voltage of $5 \mathrm{kV}$ and an ITER-relevant filling pressure of $0.30 \mathrm{~Pa}$ has been applied in b). The slightly higher pressure has been used in a) in order to lower the amount of co-extracted electrons and thus being better able to discriminate the fraction of ions in $I_{\mathrm{e}}$. Both figures show the same main features: while the drain and electron current are strongly increasing for higher RF power, the ion current shows a saturation (strong at $U_{\mathrm{ex}}=4 \mathrm{kV}$ and weak at $U_{\mathrm{ex}}=5 \mathrm{kV}$ ). In both cases, the negative ion density in the plasma has a larger slope than the extracted ion current. At higher extraction voltages $\left(U_{\mathrm{ex}} \gtrsim 9 \mathrm{kV}\right)$, a linear dependence between $I_{\mathrm{ex}}$ and $n_{\mathrm{H}^{-}}$is observed during variation of the RF power ${ }^{16,18}$. The deviation from this linear behavior is caused by an increasing fraction of negative ions scraped at the extraction grid. The scraped ions are not contributing to the measured ion current but counted as electron current.

The scraping is more pronounced at lower extraction voltage (i.e. in Figure 2 a)): although the range of RF power in this scan is smaller (37-64 $\mathrm{kW})$ compared to Figure $2 \mathrm{~b}$ ) $(28-78 \mathrm{~kW})$, the non-linear behavior between $I_{\mathrm{ex}}$ and $n_{\mathrm{H}^{-}}$is more pronounced. The co-extracted electron current at low RF power is almost zero; it should be noted that such a low amount of co-extracted electron current can only be achieved under specific circumstances (low extraction voltage, low to moderate values of RF power and a sufficient Cs conditioning); in addition, the measurement uncertainty of low currents mentioned before should be taken into account. The low coextracted electron current at low RF power means that no ions are scraped at the EG. At larger values of RF power $I_{\mathrm{e}}$ is increasing; and in this particular scan the major fraction of $I_{\mathrm{e}}$ is assumed to be scraped ions. This statement is assisted when comparing the extracted currents for different applied extraction voltages.

The same currents $\left(I_{\mathrm{drain}}, I_{\mathrm{ex}}\right.$ and $\left.I_{\mathrm{e}}\right)$ are plotted in Figure 3 as function of the extraction voltage for a constant RF power of $60 \mathrm{~kW}$. At this RF power, the extracted ion current $I_{\mathrm{ex}}$ is already strongly saturated in Figure 2 a). The same elevated source filling pressure of $0.37 \mathrm{~Pa}$ as in Figure 2 a) has been used for lowering $I_{\mathrm{e}}$; the ratio $U_{\mathrm{acc}} / U_{\mathrm{ex}}$ has been kept constant at a value of 6.5 in order to keep a similar beam optics. Whereas the extracted ion current $I_{\mathrm{ex}}$ is increasing for higher extraction voltage, the electron current decreases at higher extraction voltage; this is a counter-intuitive behavior, which can only be explained by an increased amount of negative ions scraped at the EG for lower values of the extraction volt- 


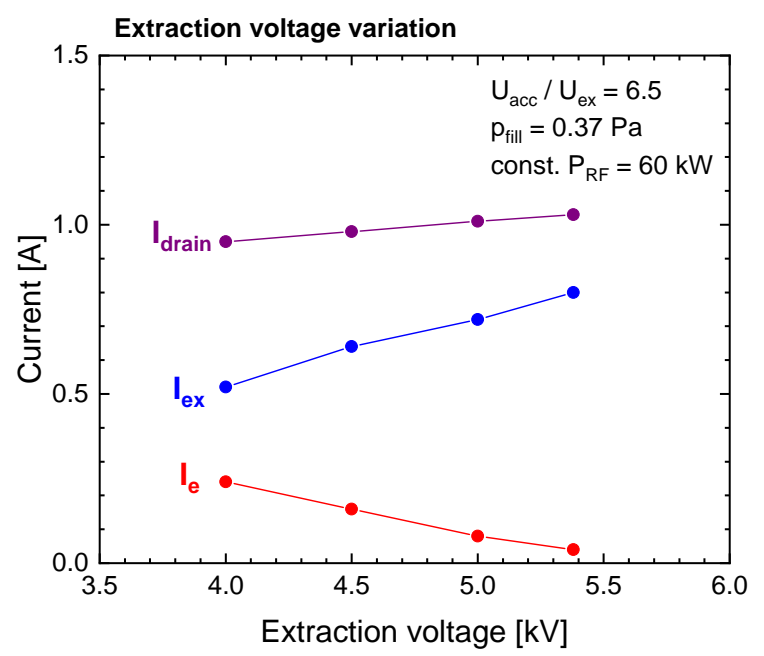

FIG. 3. Extracted currents (ion and electron) and drain current as function of the extraction voltage for a constant RF power of $60 \mathrm{~kW}$.

age. As a consequence, $I_{\mathrm{ex}}$ underestimates significantly the extracted ion current when beamlets are scraped at the EG.

Beamlet scraping at the EG is also seen in simulations modeling the BATMAN Upgrade grid geometry using the IBSimu code for low extraction voltage and reasonable high injected negative ion current densities (i.e. significant scraping for $j_{\text {injected }} \gtrsim 10 \mathrm{~mA} / \mathrm{cm}^{2}$ at $\left.U_{\mathrm{ex}}=4 \mathrm{kV}\right)^{12}$.

\section{B. Influence of Beamlet Scraping on the Divergence}

The divergence of the single beamlet (measured by the CFC calorimeter \& BES), as well as for the group of beamlets (BES) is shown in Figure 4 for the same power variation as in Figure $2 \mathrm{~b}$ ). An extraction voltage of $5 \mathrm{kV}$ and, for optimum beam optics of the individual beamlet ${ }^{8}$, an acceleration voltage of $35 \mathrm{kV}$ has been used. The resulting extracted ion current density $j_{\mathrm{ex}}$ was $9.3-13.3 \mathrm{~mA} / \mathrm{cm}^{2}$, which is normal for a medium-well conditioned source operated at these parameters. As already reported ${ }^{8}$, the diagnostics show generally a good agreement for the single beamlet and a higher divergence for the group of beamlets. The minimum beamlet divergence of $14.5-16.2 \mathrm{mrad}(\mathrm{CFC}-\mathrm{BES})$ is reached at the maximum applied RF power of $78 \mathrm{~kW}$; the minimum divergence of the grouped beamlets is $33 \mathrm{mrad}$, about a factor of two larger than for the single beamlet.

An interesting feature is that the measured divergence curve does not show an inflection point with increasing divergence for higher RF power, as would be expected: with increasing extracted ion current a transition to an overperveant regime is expected ${ }^{21}$, i.e. the divergence should increase with the RF power. However, the observed beamlet scraping at low extraction voltage and high RF power has an influence on the divergence of both the single beamlet and the group of beamlets, resulting in a reduced divergence and thus the disappearance of the inflection point in the observed range of RF power.

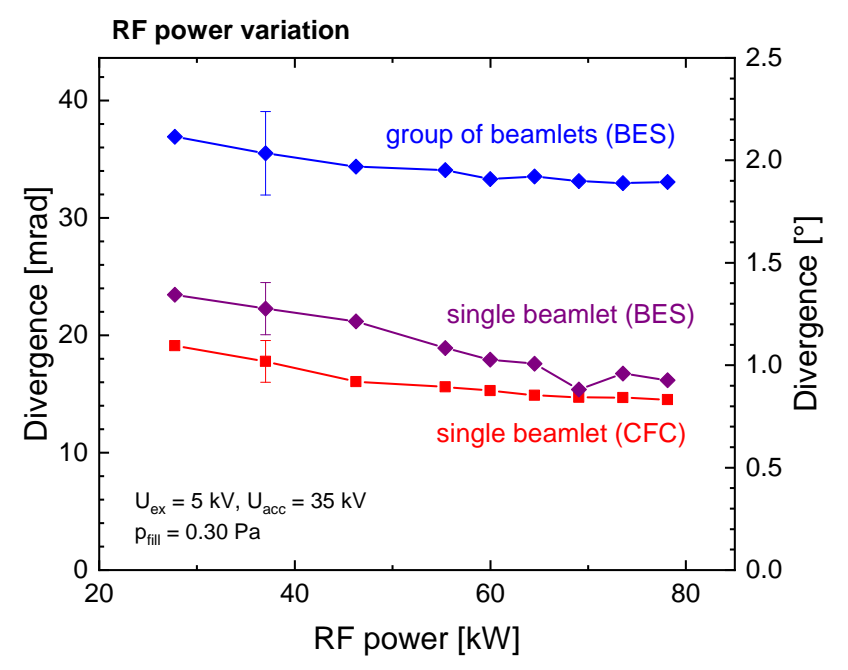

FIG. 4. Divergence as function of the applied RF power for the single beamlet (measured by BES and CFC calorimetry) and the group of beamlets (measured by BES only).

\section{CONCLUSIONS}

Under certain conditions (low extraction voltage $U_{\text {ex }} \lesssim$ $5 \mathrm{kV}$, high RF power), the negative ion beam can be significantly scraped at the extraction grid at BATMAN Upgrade. In these conditions, the measured ion current $I_{\mathrm{ex}}$ underestimates the extracted ion current, whereas the measured electron current $I_{\mathrm{e}}$ contains a fraction of negative ions. Consequently, the ratio $I_{\mathrm{e}} / I_{\mathrm{ex}}$ overestimates the real electron fraction. The beamlet scraping can be determined by deviations from the normally seen linear dependence between the extracted ion current and the density of negative ions in the plasma close to the extraction system for variations of the RF power. The beam is scraped more for lower values of extraction voltage and higher values of RF power. In case of a scraping, the determination of the beam perveance is not straight-forward because the electrical measured extracted ion current $I_{\mathrm{ex}}$ underestimates the total extracted ion current (i.e. the amount that passed the plasma grid).

Measurements showed that scraping the beam at the extraction grid lowers its divergence (beamlet and group of beamlets): in a regime at high RF power, in which an overperveant beam and thus an increase of the divergence is expected, the divergence in fact is still decreasing. The minimum value of the beam divergence (single beamlet and group of beamlets) is reached in the scraped regime at maximum RF power with a value of $14.5-16.2 \mathrm{mrad}$ for the single beamlet. Although the effect of beamlet scraping needs to be considered at low extraction voltages, there is no indication of beamlet scraping at large extraction voltage $(9-10 \mathrm{kV})$, where a linear correlation between $I_{\mathrm{ex}}$ and $n_{\mathrm{H}^{-}}$is measured ${ }^{16}$ and consequently the EG current $I_{\mathrm{e}}$ consists only of electrons. 


\section{REFERENCES}

${ }^{1}$ ITER, "Neutral beam heating and current drive system," (2011), design Description Document (DDD) 5.3.

${ }^{2}$ R. Hemsworth, H. Decamps, J. Graceffa, B. Schunke, M. Tanaka, M. Dremel, A. Tanga, H. D. Esch, F. Geli, J. Milnes, T. Inoue, D. Marcuzzi, P. Sonato, and P. Zaccaria, Nucl. Fusion 49, 045006 (2009).

${ }^{3}$ R. S. Hemsworth, D. Boilson, P. Blatchford, M. Dalla Palma, G. Chitarin, H. P. L. de Esch, F. Geli, M. Dremel, J. Graceffa, and D. Marcuzzi, New J. Phys. 19, 025005 (2017).

${ }^{4}$ K. Berkner, R. Pyle, and J. Stearns, Nucl. Fusion 15, 249 (1975).

${ }^{5}$ B. Heinemann, U. Fantz, W. Kraus, L. Schiesko, C. Wimmer, D. Wünderlich, F. Bonomo, M. Fröschle, R. Nocentini, and R. Riedl, New J. Phys. 19, 015001 (2017).

${ }^{6}$ U. Fantz, F. Bonomo, M. Fröschle, B. Heinemann, A. Hurlbatt, W. Kraus, L. Schiesko, R. Nocentini, R. Riedl, and C. Wimmer, Fusion Eng. Des. 146, 212 (2019).

${ }^{7}$ M. Bacal and M. Wada, Appl. Phys. Rev. 2, 021305 (2015).

${ }^{8}$ A. Hurlbatt, F. Bonomo, A. Pimazzoni, P. Veltri, M. Agostini, M. Barbisan, M. Brombin, R. Delogu, U. Fantz, B. Heinemann, N. den Harder, G. Orozco, C. Poggi, L. Schiesko, G. Serianni, M. Ugoletti, and C. Wimmer, submitted to Nucl. Fusion (2019).

${ }^{9}$ P. Franzen and U. Fantz, AIP Conf. Proc. 1390, 310 (2011).
${ }^{10}$ M. Barbisan, F. Bonomo, U. Fantz, and D. Wünderlich, Plasma Phys. Control. Fusion 59, 055017 (2017).

${ }^{11}$ T. Kalvas, O. Tarvainen, T. Ropponen, O. Steczkiewicz, Ärje. J., and H. Clark, Rev. Sci. Instrum. 81, $02 B 703$ (2010).

${ }^{12}$ N. den Harder, (2019), Private Communication.

${ }^{13}$ P. Veltri, (2019), Private Communication.

${ }^{14}$ G. Serianni, M. De Muri, A. Muraro, P. Veltri, F. Bonomo, G. Chitarin, R. Pasqualotto, M. Pavei, A. Rizzolo, M. Valente, P. Franzen, B. Ruf, and L. Schiesko, Rev. Sci. Instrum. 85, 02A736 (2014).

${ }^{15}$ G. Serianni, F. Bonomo, M. Brombin, V. Cervaro, G. Chitarin, S. Cristofaro, R. Delogu, M. D. Muri, D. Fasolo, N. Fonnesu, L. Franchin, P. Franzen, R. Ghiraldelli, F. Molon, A. Muraro, R. Pasqualotto, B. Ruf, L. Schiesko, M. Tollin, and P. Veltri, AIP Conf. Proc. 1655, 060007 (2015).

${ }^{16}$ C. Wimmer, U. Fantz, E. Aza, J. Jovović, W. Kraus, A. Mimo, and L. Schiesko, AIP Conference Proceedings 1869, 030021 (2017).

${ }^{17}$ B. Zaniol, J. Quant. Spectrosc. Radiat. Transfer 112, 513 (2011).

${ }^{18}$ M. Berger, U. Fantz, S. Christ-Koch, and NNBI-Team, Plasma Sources Sci. Technol. 18, 025004 (2009).

${ }^{19}$ B. Heinemann, M. Fröschle, H. D. Falter, U. Fantz, P. Franzen, W. Kraus, R. Nocentini, R. Riedl, and B. Ruf, AIP Conf. Proc. 1655, 060003 (2015).

${ }^{20}$ W. Kraus, L. Schiesko, F. Bonomo, U. Fantz, B. Heinemann, and A. Hurlbatt, AIP Conference Proceedings 2052, 040004 (2018).

${ }^{21}$ R. Maurizio, U. Fantz, F. Bonomo, and G. Serianni, Nucl. Fusion 56, 066012 (2016). 